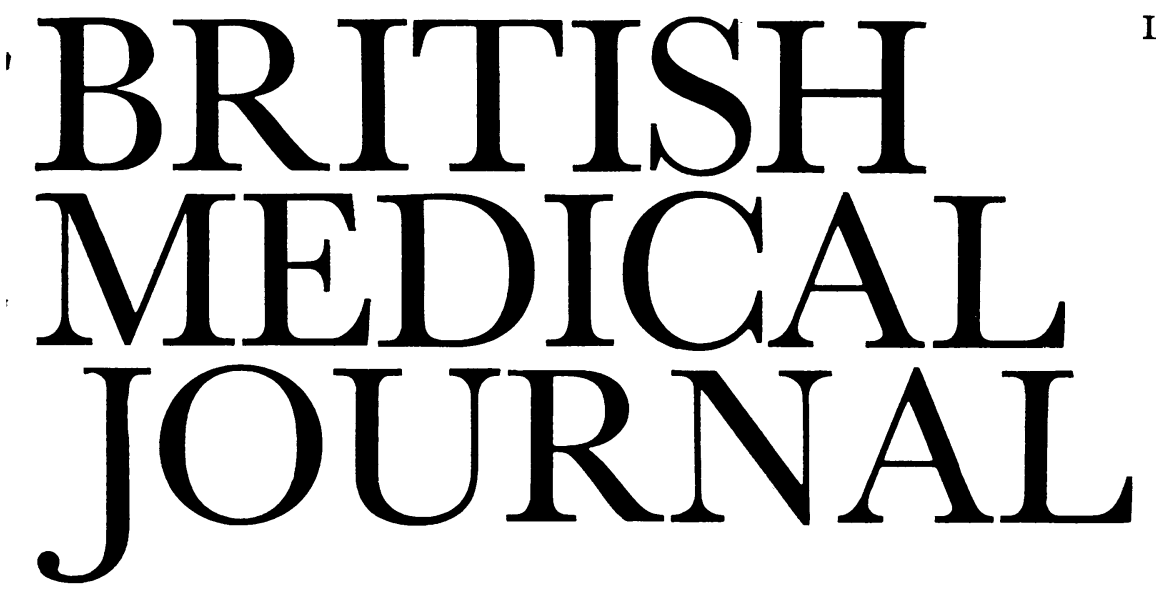

LONDON, SATURDAY 19 AUGUST 1978

\title{
Postoperative pain
}

It is an indictment of modern medicine that an apparently simple problem such as the reliable relief of postoperative pain remains largely unsolved. Numerous patients will bear testimony to our shortcomings: indeed, after talking to their friends, many will expect pain after their operation. At whose door can we lay the responsibility-the patients, the doctors, the nurses, or the pharmaceutical industry?

The patients themselves must take a fair share of the blame. In no symptom are they more inconsistent and unreliable. After identical operations under identical anaesthetics carried out by the same specialists, two patients in the same ward may apparently experience entirely different degrees of pain. Personality differences may account for some of the variations, but we certainly do not understand these well enough confidently to provide each individual with exactly what he or she needs.

No accepted method for measuring pain has yet been devised. Some workers ${ }^{1}$ prefer to use an objective assessment, in which the patient's own opinion is discounted in favour of that of the trained observer. Others ${ }^{23}$ prefer the frank subjective approach, pointing out that pain is what the patient feels and that only the patient can estimate the severity or relief.

The plethora of new parenteral agents which the pharmaceutical companies have introduced over the last 20 years is a reminder that we have not found the right drug. All, so far, have had their limitations in the form of dangerous or unpleasant side effects, or both, when given in sufficient doses to relieve severe pain. While, among the newer agents, fentanyl, phenoperidine, and pentazocine have found a valued and accepted place for analgesia during an operation, morphine (or papaveretum) and pethidine are still by far the most commonly used drugs after it. No drug seems to have appreciably exceeded the therapeutic ratio of morphine or combined its analgesic properties with a comparable degree of mental sedation and feeling of well being.

The pharmacological plot has thickened with the finding that some opiate antagonists can actually be analgesics in themselves. Of these, pentazocine, a member of the benzomorphine series, is the most widely used. It is a moderately potent analgesic comparable with pethidine but is shorter acting and does not possess the sedative effects of morphine. It does not depress the circulation in therapeutic doses and carries marginally less risk of depressing respiration or of inducing nausea or vomiting. Nevertheless, it does produce psychotomimetic side effects, which are related to the dose. These reduce its value in the prolonged treatment of very severe pain.

Meptazinol is a new opiate antagonist which produces postoperative analgesia similar to that obtained with pethidine and pentazocine. ${ }^{4}$ Its potency, milligram for milligram, is similar to pethidine's but its action and sedative effect are somewhat shorter. Cardiovascular performance is not reduced and respiration is thought to be less affected than with pethidine. The main potential advantage, however, is that in animal studies it has shown a low dependence potential. ${ }^{5}$ If these non-addictive properties are confirmed in man and further clinical trials show no psychotomimetic side effects, then meptazinol could find a place as a useful analgesic, comparable with that of pentazocine.

The latest parenteral agent to be introduced is buprenorphine, an opiate-like partial agonist-antagonist which is related to diprenorphine. It is a potent analgesic, with $0.3 \mathrm{mg}$ providing initial relief of pain similar to that with $10 \mathrm{mg}$ morphine or $75 \mathrm{mg}$ pethidine. Several papers have drawn attention to its valuable long duration of action, which may be at least twice that of morphine ${ }^{6} 7$ and four times that of pethidine. ${ }^{8}$ Drowsiness and sedation occur when the drug is used for postoperative pain relief but these are not necessarily undesirable. Respiratory depression and nausea both occur, but there is no serious depression of the cardiovascular system and some authorities have reported a rise in the heart rate and systolic arterial, central venous, and right intraventricular pressures. ${ }^{9}$ Undoubtedly these early reports indicate that buprenorphine is one of the most promising parenteral analgesic agents to be introduced in recent years.

The safety of the opiates and their derivatives has been considerably enhanced by the introduction of naloxone, which is a reliable antagonist. Unfortunately, it reverses the analgesic effects as well as the undesirable depressant actions. Nevertheless, unlike its predecessor, nalorphine, naloxone will produce no untoward effects if it is given in error to patients whose respiratory depression is not due to opiates, and it will also antagonise the action of drugs such as pentazocine.

The use of parenteral drugs is not the only method of 
relieving postoperative pain. Continuous epidural analgesia with an indwelling catheter has enjoyed periodic popularity and is extremely effective after both thoracic and abdominal operations, especially where respiratory function is precarious. ${ }^{10}$ Nevertheless, special skill is required in establishing and fixing the catheter in the correct space and in arranging for accurate top-up doses to be given safely and reliably to minimise the potentially disastrous effects of infection and overdose.

Current inhalational agents have only a limited place in postoperative analgesia. The safe self-administration of Entonox (oxygen and nitrous oxide) cannot usually be employed for long periods but is invaluable where short, sharp bouts of pain are expected, such as occur during physiotherapy, change of dressings, removal of drains, ${ }^{11}$ and helping defecation after haemorrhoidectomy. Nitrous oxide should also not be forgotten as a safe analgesic for patients on mechanical ventilators, particularly in those whose circulation is in jeopardy. It is, after all, still the basis of safe anaesthesia.

In clinical practice, pain relief after operation is largely unsupervised by doctors, who merely write the prescription on an "as required after a certain interval" basis. This often means that the patient has to take the initiative in asking for analgesia. Nevertheless, though most wait until their pain is severe in order not to appear cowardly or demanding, there is considerable evidence that pain is more easily controlled with smaller doses of drugs if it is treated early before becoming severe. Indeed, both Kamel and $\mathrm{Geddes}^{8}$ and $\mathrm{Kay}^{7}$ have suggested that the best results may be achieved if all patients liable to experience postoperative pain are given an analgesic intravenously by the anaesthetist at the end of their operation.

In the later postoperative period most of the parenteral analgesics in common use are more effective and safe if given in moderate doses every two to three hours rather than the traditional larger dose every six hours. The intravenous route is better than the intramuscular one, from which uptake is notoriously unreliable, especially in the ill patient. Working in obstetric analgesia, Rosen ${ }^{12}$ has shown that pain can be well controlled with predetermined increments of pethidine or other opiates which the patient gives herself using a preloaded mechanical syringe attached to an intravenous catheter. This technique can also be successfully applied to relieve postoperative pain after upper abdominal surgery and takes account of biological variation in pain tolerance. Certainly we still have a lot to learn about ensuring that our patients have as little pain after operation as possible.

1 Parkhouse, J, and Holmes, C M, Proceedings of the Royal Society of Medicine, 1963, 56, 579.

2 Beecher, H K, Measurement of Subjective Responses. New York, Oxford University Press, 1959.

3 Huskisson, E C, Lancet, 1974, 2, 1127.

4 Paymaster, N J, British fournal of Anaesthesia, 1977, 49, 1139.

5 Goode, P G, and White, A C, British Fournal of Pharmacology, 1971, 43, 462 .

6 Dobkin, A B, Esposito, B, and Philbin, C, Canadian Anaesthetists' Society Fournal, 1977, 24, 195.

7 Kay, B, British fournal of Anaesthesia, 1978, 50, 605.

8 Kamel, M M, and Geddes, I C, British fournal of Anaesthesia, 1978, 50, 599.

- De Castro, J, and Parmenter, $\mathrm{P}$, in Proceedings of the VI World Congress on Anaesthesiology, section 55, vol 7, ed L Lecron, p 103. Charleroi, Société d'Anésthésie, 1976.

10 Griffiths, D P G, Diamond, A W, and Cameron, J D, British fournal of Anaesthesia, 1975, 47, 48.

11 Baskett, P J F, and Bennett, J A, British Medical fournal, 1971, 2, 509.

12 Rosen, M, in Pain-New Perspectives in Measurement and Management, ed A W Harcus, R Smith, and B Whittle. Edinburgh and London, Churchill Livingstone, 1977.

\section{Chronic spinal arachnoiditis}

Chronic adhesive spinal arachnoiditis may be caused by infections, irritation, trauma, or rarely allergy and so its clinical, radiological, and pathological features are correspondingly varied. The essential abnormality is thickening and opacity of the arachnoid, which generally becomes adherent to the dura mater, nerve roots, or pia. The subarachnoid space may become obliterated by a dense felt of connective tissue with small round cell infiltration and vascularisation, or strands of thick arachnoid may divide the space into a labyrinth with sponge-like cavities that may become loculated to form cysts. Nerve roots may be compressed or rendered ischaemic, and the spinal cord may show ischaemic changes-at first these are usually peripheral but may progress to softening of the central part of the cord and cavitation. The cerebrospinal fluid protein concentration is usually, but not inevitably, raised, depending on the degree of obstruction and the activity of the process. These changes are irreversible but not inevitably progressive, as used to be thought: they do not respond to steroids or radiotherapy, and surgical decompression or division of adhesions confers no certain benefit.

Spinal arachnoiditis was recognised at the beginning of the century and documented again in the 1930s by Elkington. ${ }^{12}$ Its presentation and clinical features have changed over the years. Syphilis and tuberculous meningitis have disappeared, while the increased use of radiological contrast media and spinal injections of various kinds has altered the predominant location from cervicothoracic to lumbar. The sequel of myelomalacia and paraplegia is now comparatively rare. This changing pattern was apparent in the review by Shaw et $a l^{3}$ of 80 cases related to 7600 spinal contrast examinations over 21 years. Intrathecal contrast media are not, however, the only agents that nowadays may lead to this distressing condition, for it may complicate treatment with epidural steroids and other spinal injections, ${ }^{4}$ and occur as a sequel to disc lesions ${ }^{5}$ or trauma, including surgical operation.

The clinical presentation is usually with radicular pain affecting more than one segment, often bilateral. The pain is little affected by movement and straining. Stinging or burning symptoms and signs may develop immediately after the damage to the arachnoid or there may be a delay of 20 years or more-perhaps until a second episode of damage. Chronic arachnoiditis often causes no symptoms at all and may be an incidental finding, but occasionally cord symptoms appear early.

The radiological appearances include obliteration of nerve root sleeves and narrowing of the subarachnoid space. There may be a partial or complete spinal block and irregular distribution and loculation or fixity of the contrast medium. Cysts may be present, and these may or may not contain contrast or communicate with the cerebrospinal fluid. The clinical picture of chronic arachnoiditis may resemble that of a spinal tumour, and it is often necessary to exclude this possibility. In those patients who prove to have arachnoiditis the addition of further contrast medium after an earlier injection increases the risk of subsequent adhesive changes; but arachnoiditis may mask an underlying lesion, such as a protruded disc.

Clearly the risks of any investigation or treatment always have to be balanced against the probable benefits, and must be judged in the context of the facilities available. A comparative study ${ }^{6}$ of contrast media used for spinal studies has 